\title{
Physical Human-Robot Collaboration: Robotic Systems, Learning Methods, Collaborative Strategies, Sensors and Actuators
}

\author{
Uchenna Emeoha Ogenyi, Student Member, IEEE, Jinguo Liu, Senior Member, IEEE, Chenguang Yang, Senior \\ Member, IEEE, Zhaojie Ju, Senior Member, IEEE and Honghai Liu, Senior Member, IEEE
}

\begin{abstract}
This paper presents a state-of-the-art survey on robotic systems, sensors, actuators and collaborative strategies for Physical Human-Robot Collaboration (pHRC). The paper starts with an overview of some robotic systems with cuttingedge technologies (sensors and actuators) suitable for pHRC operations and the intelligent assist devices employed in pHRC. Sensors being among the essential components to establish communication between a human and a robotic system are surveyed. The sensor supplies the signal needed to drive the robotic actuators. The survey reveals that the design of new generation collaborative robots and other intelligent robotic systems has paved the way for sophisticated learning techniques and control algorithms to be deployed in pHRC. Furthermore, it revealed relevant components needed to be considered for effective pHRC to be accomplished. Finally, a discussion of the major advances made, some research directions, and future challenges are presented.
\end{abstract}

Index Terms-Robotic Systems, Sensors, Actuators, Collaborative Robots, Physical Human-Robot Collaboration, HumanRobot Collaboration, Human-Robot Interaction.

\section{INTRODUCTION}

$\mathbf{F}$ OR decades, robots have been employed in various areas of human endeavours. A robot can operate either in autonomous or collaborative modes. In the autonomous mode, the operational process often requires little or no human support. However, this mode of operation limits the applicability of a robot to certain domains of human endeavours as it is challenging for a robot to accomplish assigned task alone in a real-world environment, which is full of uncertainties and, dynamic in nature [1]. Besides the autonomous mode of

This work was supported by the National Key Research and Development Program of China (grant no. 2018YFB1304600), the Tertiary Education Trust Fund (TETFund), Nigeria, the DREAM project of EU FP7-ICT (grant no. 611391), Natural Science Foundation of China (grant no. 51575412, 51575338, 51775541, 51575407), and the Chinese Academy of Sciences Interdisciplinary Innovation Team (grant no. JCTD-2018-11).(Corresponding author: Zhaojie Ju)

U. Ogenyi and H. Liu are with the School of Computing, University of Portsmouth, Portsmouth PO1 3HE, U.K. (e-mail: uchenna.ogenyi@ port.ac.uk; honghai.liu@port.ac.uk).

J. Liu is with the State Key Laboratory of Robotics, Shenyang Institute of Automation, Institutes for Robotics and Intelligent Manufacturing, Chinese Academy of Sciences, Shenyang 110016, China (e-mail: liujinguo@sia.cn).

C. Yang is with Bristol Robotics Laboratory, University of the West of England, Bristol, BS16 1QY, UK. (cyang@ieee.org).

$\mathrm{Z}$. Ju is with the School of Computing, University of Portsmouth, Portsmouth PO1 3HE, U.K., and also State Key Laboratory of Robotics, Shenyang Institute of Automation, Institutes for Robotics and Intelligent Manufacturing, Chinese Academy of Sciences, Shenyang 110016, China (email:zhaojie.ju@port.ac.uk). operation, a robot can collaborate with humans physically or remotely to perform a task. The collaboration perhaps includes shared knowledge, experience, and belief about the task goal to be achieved [2].

By collaborating, human dexterity and flexibility are combined with the repeatability and precision of a robot which result in reduced human workload, increased productivity and applicability of robots in more areas of human endeavours [3], [4]. The collaboration could be seen in human-exoskeleton skill transfer where the exoskeleton may be required to replicate the human operator's arm impedance performance or to compensate external disturbance. Some recently conducted works to investigate human-exoskeleton skill transfer capable of achieving desirable performance both in parametrised and unparametrised circumstances are discussed in [5] and [6]. These kinds of robots are conventionally called collaborative robots and are built with unique technologies to ensure compliances with the International Organization for Standardization (ISO) specifications for designing and manufacturing collaborative robots [7]-[9].

To achieve intuitive physical human-robot collaboration (pHRC), a robot must be able to observe its surrounding in order to take cognizance and continuous update of the current state of its surroundings. With such information in place, a robot could be endowed with the ability to estimate desired actions to be performed and the best possible way to perform them. For instance, a robot collaborating with a human in a table lifting task is expected to identify the items in the environment, including the table, predict human future intention and coordinate its activities towards successful completion of the task. These capabilities cannot be naturally endowed in the robot directly from the manufacturers but could be learned through the appropriate learning method. In recent years, robots are increasingly being taught predominantly using machine learning techniques. This partially solves the dimensionality problem and the computational challenge of actively coding all the steps required for every collaborative action. However, after a task is learned, the issue of optimal implementation of the task follows. But with proper task planning and effective collaborative strategies, a robot can effectively collaborate with a human to accomplish a desired goal [10].

All these functionalities could not be possible in the absence of the state-of-the-art technologies especially, the sensors and actuators. In robotics, sensors serve as channels through which 
a robot can acquire knowledge about their surroundings, communicate contents and give feedback to the environment or users. On the other hand, actuators convert the control signals into motions in the relative joints and body of the robots (e.g. soft robotic systems). Hence, these technologies either embedded or physically attached are the hallmarks of any robotic systems suitable for effective pHRC. More details of these two important components are further discussed in Section III of the paper.

Several scholars have carried out a survey to describe the synergy that could exist between human and robot in a view to accomplish a task [11]. In [12] Chandrasekaran et al. presented a survey on Human-Robot Collaboration (HRC), however, little attention was paid on the collaborative strategies and sensing technologies involved. Further work has addressed the sensing and collaborative strategies [13], [14], but both works focused on the safety interaction between human and robots in an industrial setting. Furthermore, Ajoudani et al. work reported the control strategies, interaction modalities and collaborative interfaces for pHRC [15]. Although, these scholars made efforts to present an up-to-date overview on HRC, but to the best of the authors' knowledge, none of them covered exhaustively the most relevant challenges due to the fast-growing trends in the pHRC technologies and the tremendous demands in the applicability of robotic systems in our day-to-day activities.

In these regards, an updated overview of the state-of-theart survey on $\mathrm{pHRC}$ is presented. The paper presents a comprehensive review of the state-of-the-art robotic systems with cutting-edge technologies, such as sensors and actuators for pHRC. The collaborative strategies and the learning methods useful for $\mathrm{pHRC}$ are also discussed.

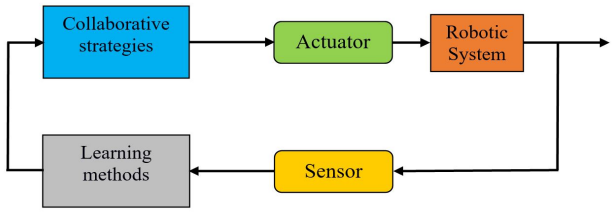

Fig. 1: A block diagram to describe how collaborative strategies relate to the learning methods, and both the sensors and actuators, and the robotic system.

Figure 1, depicts that for an effective pHRC to be implemented, relevant components such as sensors, actuators, suitable robotic system, appropriate collaborative strategies, and learning methods need to be carefully considered and chosen.

The rest of this paper is organized as follows: Section II provides a highlight of the robotic systems and other intelligent assist devices for pHRC. In section III, different kinds of sensors and actuators used in pHRC are discussed. Section IV concentrates on the formulation of actions, task planning, and other collaborative strategies. Robot learning methodologies in different collaborative scenarios are discussed in Section V. Safety issues, control designs and human factors are presented and discussed in Section VI while discussion and conclusion are presented in Section VII.

\section{Robotic Systems}

The robotic systems discussed herein are grouped based on their application purposes, prominent mechanical structures, and adaptive features. However, we acknowledge the possibilities of overlap of the robotic system, making it possible to have a robotic assist device that is also a wearable robotic system.

\section{A. Collaborative Robotic Arms}

The collaborative robot can work safely in a shared space with humans. In addition, they are power, and force limited, compact, lightweight, dexterous, and majority could be hand guided through a path by the user in order to accomplish a task, hence allowing users with little or no knowledge of programming to use them. Various prominent collaborative robots with cutting edge technologies suitable for pHRC operations are presented in this section. They are grouped into single-arm (see Fig. 2) and dual-arm ( see Fig. 3) for clarity and better understanding, and based on their physical features and areas of application. Table I and II present unique features found in single-arms and dual-arms collaborative robots respectively. Apart from the physical and structural difference between the single-arm and the dual-arm collaborative robots, there are some salient global features which serve as strengths of each group. For instance, the single-arm robots are faster and highly efficient in carrying out their tasks. They are accurate and precise, flexible deployment and fast set-up procedures. While the dual-arm robots provide users with human-like control over their environments, multiple simultaneous tasks, and synchronized motion which enables safe handling of larger and heavier parts.

1) Single-Arm Robots: Sawyer is a product of Rethink Robotic and was manufactured while targeting on high precision tasks. Sawyer comes with Intera Studio which is a graphical user interface (GUI) that allows users with limited technical skills to program the robot. Other unique features include that it is faster, lighter and more precise than Baxter (see Section IIA2). Having been designed to manoeuvre in a tight space or occupy a small space, Sawyer can perform more tasks and stand the chance of being a better candidate for the industries.

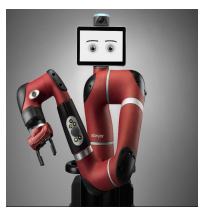

(a)

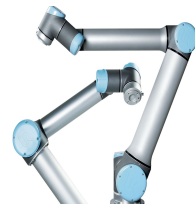

(b)

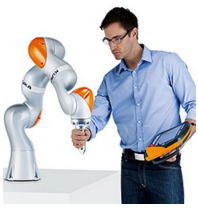

(c)

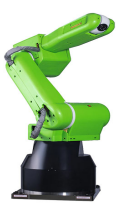

(d)
Fig. 2: The figures show single-arm collaborative robots: (a) Sawyer [16], (b) Universal [17], (c) KUKA 7 R800 [18] and (d) Fanuc-CR35ia [19].

Universal Robots (UR) came up with 3 sets of 6-DOF collaborative robots namely UR3, UR5, and UR10. The working ranges for the UR3, UR5 and UR10 are $50 \mathrm{~mm}, 850 \mathrm{~mm}$ and $1300 \mathrm{~mm}$ respectively. This set of UR robots are known for 
low noise when in production, easy to handle, adaptive and easy to customize the end-effector.

TABLE I: The single-arm robots and their unique features

\begin{tabular}{llll}
\hline Robot & DOF & Rep(mm) & Payload(kg) \\
\hline Sawyer & 7 & \pm 0.1 & 4 \\
UR3, UR5, UR10 & 6 & \pm 0.1 & $3,5,10$ \\
KUKA LBR 7R800 & 7 & \pm 0.1 & 7 \\
Fanuc RC-35iA & 4 & \pm 0.08 & 35 \\
\hline Robot & Simulator & Actuator & R/C \\
\hline Sawyer & Moveit, Gazebo & SEAs & R/C \\
& \& V-REP & & \\
UR3, UR5, UR10 & RoboDK, V-REP & SEAs & R/C \\
KUKA LBR 7R800 & Moveit \& Polyscope & & \\
Fanuc RC-35iA & ROBOGUIDE & SEAs & R/C \\
\hline
\end{tabular}

Rep $=$ Repeatability, R/C = Research and Commercial Oriented, and $\mathrm{C}=$ Commercial Oriented

KUKA, a German robot manufacturer also joined the league of collaborative robot manufacturers and came up with a lightweight collaborative robot called LBR IIWA 7-R800 [3]. Although, KUKA has excellent power to weight ratio, equipped with unique sensors that detect micro impact at the joints; however, it is quite expensive, and that might discourage buyers.

Fanuc RC-35iA is known as the strongest collaborative robot; it weighs $990 \mathrm{~kg}$ and can operate at a maximum payload of $35 \mathrm{~kg}$. Fanuc is good for heavy industrial applications, machine tending, and automation services. It is dexterous, compact but lacks the teaching by demonstration feature and has no research version. Some of these missing features perhaps can discourage potential users from acquiring the robot.

2) Dual-Arm Robots: Baxter makes use of the same operating system and end-effector design interface with the Sawyer robot. It has a very nice looking structure suitable for handling and manipulating objects simultaneously, however, it lacks a good precision when compared with Sawyer (see Section IIA1). It also occupies more space, making it less attractive to the industries.

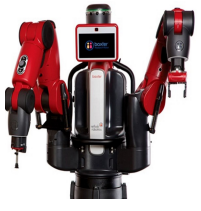

(a)

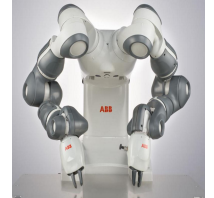

(b)

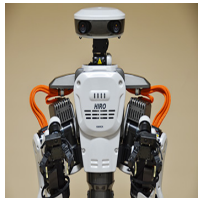

(c)

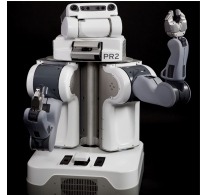

(d)
Fig. 3: The figures show dual-arm collaborative robots: (a) Baxter robot [16], (b) Yumi [20], (c) NEXTAGE Open [21] and (d) PR2 [22].

Yumi was developed by ABB Robotic and the target is for small part assembly and for testing and packaging operations in the industries [23]. Yumi is a low-cost adaptable robot. It is also flexible, easy to program, and very sensitive to outside forces. Furthermore, its compact and small-sized appearance makes it portable and suitable for many industries and research institutions.
NEXTAGE has 6-DOF per arm. It weighs $29 \mathrm{~kg}$ and can support a maximum of $1.5 \mathrm{~kg}$ payload on each arm. It is also compatible with ROS. Other interesting features include low power motors at its joints, making it safer if it collides with an object or a human, and the ability to perform complex tasks and fine manipulation due to the high DOFs of the rotating axes at its neck and waist. However, it is only sold in the Asian market.

The PR2 which is purposely for research is equipped with a lot of sensors including fingertip pressure sensors and accelerometers at the end-effectors. The PR2 is also equipped with a laser-based scan rangefinder [24] and is compatible with ROS [25]. Considering the shape and size, PR2 is suitable for both small-sized and narrow-spaced research labs; it is also mobile and could easily be moved from one lab to another.

TABLE II: The dual-arm robots and their unique features

\begin{tabular}{llll}
\hline Robot & DOF & Rep(mm) & Payload(kg) \\
\hline Baxter & 7 & \pm 0.1 & 2.2 \\
Yumi & 7 & \pm 0.2 & 0.5 \\
NEXTAGE & 6 & \pm 0.03 & 1.5 \\
PR2 & 4 & \pm N/A & 1.8 \\
\hline Robot & Simulator & Actuator & R/C \\
\hline Baxter & Moveit, Gazebo & SEAs & R/C \\
& \& V-REP & & \\
Yumi & RoboDK, V-REP & SEAs & R/C \\
& Moveit \& Polyscope & & \\
NEXTAGE & RoboDK, V-REP & SEAs & R/C \\
& Moveit \& Polyscope & & \\
PR2 & RoboDK, V-REP & SEAs & R \\
& Moveit \& Polyscope & & \\
\hline
\end{tabular}

Rep = Repeatability, R/C = Research and Commercial Oriented $\mathrm{C}=$ Commercial Oriented and $\mathrm{R}=$ Research Oriented

\section{B. Wearable Robotic Systems}

Wearable robotic systems are currently employed in different areas including to improve a damaged body function or to enhance the physical abilities.

1) Upper-Limb Wearable Robotic Systems: Different types of upper-limb wearable robotic arms have been proposed and designed by various researchers. Some notable wearable upper-limb robotic arms and their unique features are presented in Table III.

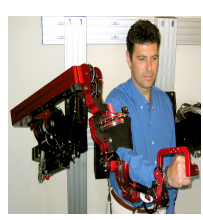

(a)

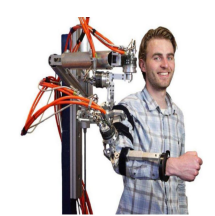

(b)

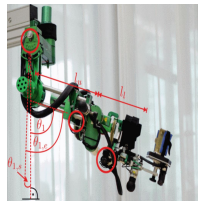

(c)

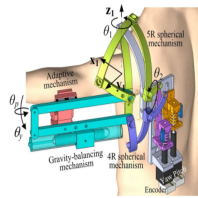

(d)
Fig. 4: The wearable robotic arms (a) EXO-UL7 [26], (b) LIMPACT [27], (c) ARMin-V [28] and (d) A CAD model of the parallel actuated exoskeleton [29].

University of California Los Angeles (UCLA), Bionic Lab developed exoskeleton robot (EXO-UL7) which has the same 
DOF as a human hand [30]. In order to support humanmachine interaction, the robot is equipped with force/torque sensors on the upper arm, the lower arm, the hand and on the tips [26]. The operation of this device is based on a muscular signal generated from the human-machine interface (bioport) using electromyography (EMG). Similarly, Zhijun et al. proposed an asymmetric bimanual coordinate control for the dual-arm exoskeleton to perform human-cooperative manipulation [31]. The contribution of the work focused on handling physical constraints such as joint limits and torque limits via the use of human motion intention reflected during interaction and the use of impedance parameters approximation for estimating variable stiffness which measures the force and position of the dual-arm end-effector.

The major aim of developing LIMPACT is for reflex identification on the arm of a stroke survivor. The identified reflex could be used to design an optimised program therapy for stroke survivors [27]. The LIMPACT has a torque controlled motors with a maximum of $79 \mathrm{~Hz}$ bandwidth which allows for smooth zero impedance control. The actuation mechanism in LIMPACT is based on hydraulics powered by an electric motor. LIMPACT is equipped with a passive weight balancing mechanism to compensate for the heavy load, usually experienced in the exoskeleton, and to maintain a smooth trajectory.

The ARMin-V is a 7-DOF exoskeleton robot that is actuated by direct current (DC) motor [32]. The earlier version was proposed for rehabilitation of stroke patient; but, does not incorporate online adaptive compensation. This feature is the major contribution of ARMin- $\mathrm{V}$ and it increased the performance of the robot to the level of adjusting patient's anthropometry automatically [28]. Another work in [33] presents a framework for adaptive admittance control by incorporating and considering human motion intention in order to perform more accurately in the actual physical interaction. The framework consists of double control loops with the inner loop overseeing the unknown masses and inertia of the robot dynamics while the outer loop harmonies the interaction based on the observed intention of the subject.

A parallel actuated exoskeleton was proposed in [29] to provide after stroke rehabilitation. The system is a 6-DOF exoskeleton which comes with two linear SEA for interactive force control and two gravity mechanisms to increase the device compatibility and minimize the load of the motor [29]. Hsieh et al. claim that using SEA reduces the impedance of the exoskeleton more than what the result of using feedback from pressure and force sensors could do [34].

TABLE III: The upper-limb wearable robotic arms

\begin{tabular}{llll}
\hline Robot & DOF & Actuator & R/C \\
\hline EXO-UL7 & 7 & DC motors & $\mathrm{R}$ \\
LIMPACT & N/A & Hydraulic & R/C \\
ARMin-V & 7 & Electric motor & R \\
{$[29]$} & 6 & SEAs & R/C \\
HEXAR & 6 & Electric motor & $\mathrm{R}$ \\
\hline R/C = Research and Commercial Oriented, and & & & \\
R = Research Oriented & & &
\end{tabular}

2) Lower-Limb Wearable Robotic Systems: Several lowerlimp wearable robotic systems have been developed in the past for rehabilitation and enhancement of the strength of the lowerlimb to help in carrying heavy loads. Aguilar designed a hybrid actuated lower-limb wearable robot for force augmentation and cyclic rehabilitation [35] (see Fig.5 a). The lower-limb wearable robotic systems have also received great attention in the study to minimize the metabolic cost by using the powered ankle-foot orthosis [36]-[38]. Furthermore, Collins et, al. developed a recent ankle exoskeleton device which has been able to lower metabolic cost by $7.2 \%$ [39]. This came after the breakthrough of the one developed by the Massachusetts Institute of Technology (MIT) which led to an $8 \%$ reduction in the metabolic cost of the user [40] (see Fig.5 b). Another, authors developed a human-cooperative control exoskeleton for human locomotion assistance in climbing stairs without clutches [41]. This is achieved by the adaptive controller designed to simultaneously incorporate human and robot's capabilities.

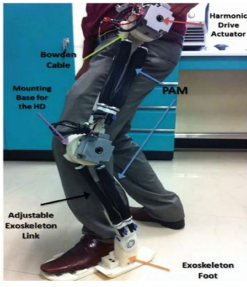

(a)

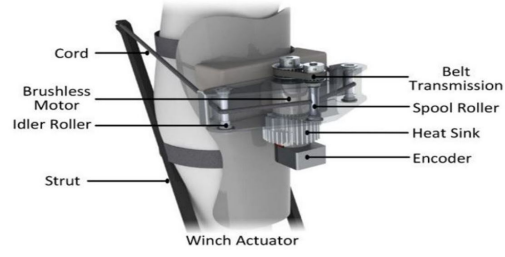

(b)
Fig. 5: (a) The system uses a hybrid of pneumatic-electric system with a harmonic drive actuator to enhance its strength [35], (b) The autonomous leg exoskeleton consists of a winch actuator and fiberglass struct that directly apply a resultant torque about the human ankle joint during walking [40].

The lower-limb wearable robotic systems are often used interchangeably with the walking assist devices (see Section IIC2), however the former mainly aim at supporting healthy people and for lower-limb rehabilitation while the latter aims at supporting elderly people or paraplegics. Furthermore, the lower-limb wearable robotic systems are mainly employed to enhance the strength of healthy people in performing heavy tasks, like in carrying heavy loads, and as a therapy for lowerlimb movement rehabilitation, like in a stroke patient. While the walking assist devices are mainly employed to reduce the load on human legs and provide motion support necessary to generate the force needed to perform the intended stride.

3) Prosthetics: In recent years, both the upper-extremity and lower-extremity have received a great attention from researchers [42]. Despite, the success of prosthesis, Kairu et al. [42] and [43] noted that most common commercial prostheses can not support users in performing daily living activities such as grasping and holding onto an object without slip, due to either the absence of perceptual tactile feedback or no tactile feedback at all. It was recorded that by measuring the characteristics of touch, tactile sensing can improve the ability of amputee to achieve a stable grasp and prevent slip [44], [45]. To extend on that, various methods were proposed to sense slip in the prosthesis. Masuda et al. proposed object displacement measurement to detect when slip is obvious [46]. 
However, it is hard to determine the minimum displacement required before a slip can occur. Hence the object could be lost from the hand before appropriate actions could be taken to prevent slip. Alternatively, sensing vibration on the hand can be an indication that a slip is about to occur and an accurate estimation of friction coefficient can help determine the possibility of slip occurring [47].

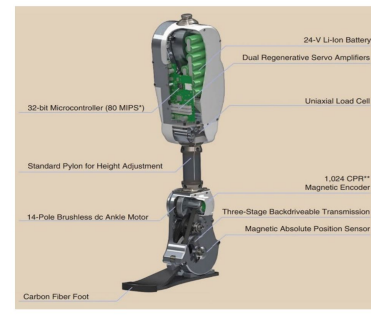

(a)

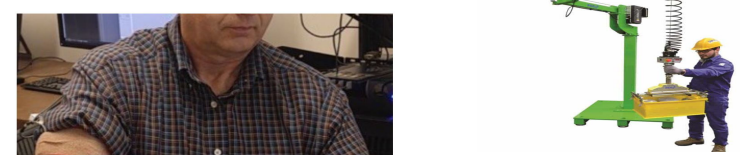

(a)

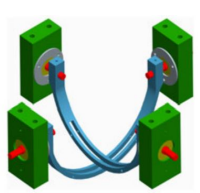

(b)

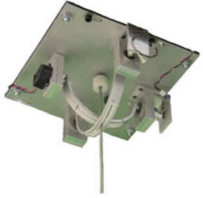

(c)
Fig. 7: (a) A handle controlled IAD for load lifting assistance, (b) and (c) depict a CAD model of the cable angle sensor and a prototype of the cable angle sensor respectively [55].

In most IADs, the operator manipulates the system through an instrumented control handle which could be equipped with force sensors to measure the force applied on the load by the operator [54]. However, the force is affected by the dynamical effects of the payload. This must be accounted for, in order to properly estimate the force needed to effectively move the payload around. Based on that, the cable angle sensor [55], (see Fig.7 b and c) was introduced by researchers to deal with this challenge. The suspended cable (see Fig.7 c) is passed through the concentric groove parts in order to drive them individually as it moves along one of the concentric parts. Each concentric part is attached to a shaft and the cable angles are obtained by measuring the shaft rotation. The authors claim that the effect of dynamics on the cable is negligible because the design makes moving the payload lighter and smoother. Despite the research progress, there is still demand for a researchable solution, as it is still challenging to find the most appropriate way to measure the inclination of the cable sensor.

\section{SENSORS AND ACTUATORS} the user. The MuscleSuits (INNOPHYS CO., LTD) which is powered by compressed air and Hybrid Assistive Limb (HAL) exoskeleton suits produced by CYBERDYNE INC., perform similar functions as AWN-03 [51].

2) Walking Assist Devices (WADs): This was developed to support body weight and reduce the load on human legs while walking. In 2009, Honda published a paper detailing the working principles of their WAD first product. The control mechanism of the WAD is based on finding a balancing point between the target assist desired force of the user with the generated force feedback from the force sensors attached to the foot arches of the WAD [52]. Similarly, the WalkON Suit was designed to assist paraplegics to walk and exercise [53]. The control technology is based on a hybrid actuation mechanism and a biarticular transmission system.

3) Intelligent Assist Devices (IADs): Tan et al. described the IAD as an intelligent machine that optimizes moving and lifting operations by reducing the physical force involvement of a human partner [54]. Compared to the traditional lifting manipulators, IAD is better because it is easier to operate, safer and allows for easy control of the payload motion (see Fig.7 a).

\section{A. Robotic Sensors}

1) Vision-Based Sensors: Recently, camera sensors are mostly used in pHRC to observe the environment because they are convenient, relatively cheap and easy to use. A camera sensor can provide RGB information and depth information for robot utilization. The Kinect sensor is a popular camera sensor that is designed to provide RGB-D information at the rate of $30 \mathrm{~Hz}$. It can also provide human tracking API [56], which works effectively even in real-time [57]. Kinect sensor has been significantly utilised in [58], [59] and [60] to track human body motion that is employed for robot control. The limitation of a camera sensor is that it is prone to occlusion and suffers from light conditions such as reflection and contrast.

2) Robotic Skin: A lot of attention has been paid on classifying sensing technologies based on their sensing principles and designed technologies such as resistive, capacitive, piezoelectric, acoustic and so on; for a more detailed review see [61], [62]. However, the design could be done differently by paying attention to the tactile information processing. One interesting and useful sensor that has moved towards this 
direction is the robotic skin. The technology provides rich and direct feedback that enables robotic systems to identify objects via multiple contact points.

- Optical Skin-Based Sensors: The general idea of optical skin-based sensor is to develop a multi-modal sensor capable of providing both tactile and visual information. Inline with that, some researchers proposed to cover the skin surface of the sensor with an opaque material meant to shut out external light from entering the sensor. However, the use of an opaque material limits the information provided by the visual sensor; hence making them to focus only on the tactile information. To address this, a prototype consisting of transparent skin, cameras, and the coloured markers was proposed in [63]. The proposed sensing skin gives a high resolution of contact force and proximity vision. The markers are used to track skin deformation which is proportional to the displacement caused by the external force. The cameras lens are focused on the markers as shown in Fig. 8 to improve the markers tracking quality. The authors discovered that the tracked skin deformed information could be used for contact force and torque estimation [64].

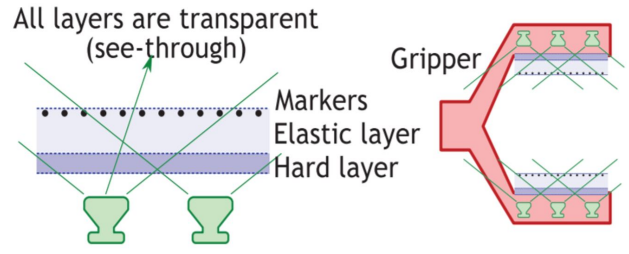

Fig. 8: A conceptual design of the optical skin-based sensor as it is installed on a robotic gripper.

- Soft Skin-Based Sensors: A wide variety of conventional flexible sensors are faced with technical issues that reduce the sensitivity of the sensor. For example, aging and mechanical stress can lead to hysteresis and reduction in the sensitivity of the sensor [65], [66].

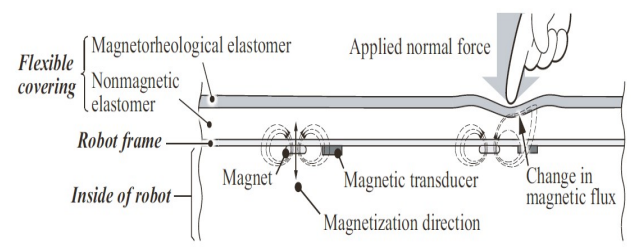

Fig. 9: A certain amount of magnetic flux generated by the magnet penetrates the elastomer as shown in the left section of the figure, however in the presence of contact force, the magnetic flux penetration increases because of a decrease in the distance between the magnet and the transducer as shown in the right section of the figure.

To address this challenge, Kawasetsu et al. proposed a magnetorheological tactile sensor consisting of a flexible upper and lower layers elastomer [67]. A deformation on the elastomer sheet causes a change in the magnetic flux which affects the spatial response of the sensor.
This is because the applied force (see Fig. 9) causes a decrease in the distance between the upper layer and the transducer which in turn increases the level of magnetic flux penetrating the transducer.

In a similar work, Kaboli et al. developed an in-hand object exploration tactile descriptor to extract robust tactile information from generated vibrotactile signal [68]. The proposed learning transfer algorithm works by first measuring the dynamic pressure signal at a sample rate of $2 \mathrm{KHz}$ and secondly the Shadow Hand via the impedancesensing electrodes which explores the texture of the object at a sample rate of $50 \mathrm{KHz}$. Then the impedancesensing electrodes enable the deformation on the skin surface to be measured. The measured deformation amounts to the force applied on the surface of the skin. The drawback is that the vibrotactile signal is prone to noise and filtering it out is computationally expensive. In another work, a set of novel tactile descriptors for multimodal robotic skin (see Fig.10) to discriminate among objects and material via their textural properties was proposed in [69]. Their tactile descriptors considered the statistical properties of the tactile signals both in stationary and dynamic states. Hence, making it invariant with respect to exploratory movement and time.

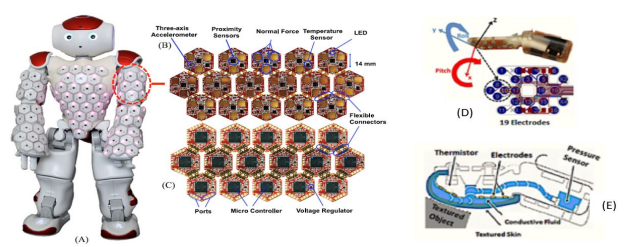

Fig. 10: (A) NAO equipped with the sensor on the chest, the fore and upper arms. (B) and (C) show the front and back views of the multi-sensing devices, connectors, voltage regulator, ports and Micro controllers.

3) Electromyography (EMG) and Electroencephalography $(E E G)$ : Electromyography (EMG) sensor is used to record the activities of electrical signals that are generated when a muscle contracts. Information from the systems could be used to estimate human limb motion [70], [71] which is essential for the reconstruction of robotic prosthetic for amputees and also important for limb rehabilitation [72], [73]. This system has been applied to detect hand grasping actions in [74] and in [75] to transfer writing skill from a human to a robot.

Electroencephalography (EEG) signal has been used purposely for communication between the human brain and a computer system, to diagnose epilepsy [76] and other brain disorders [77]. Currently, this signal capability is extended to establish communication between the human brain and a robotic system [78], and to send a control signal from a human to a robotic system [79].

4) Data Gloves: A data glove can provide information about a human hand motion, hand pose and orientation in $3 \mathrm{D}$ space [80] and even force information [81]. The application of enhanced sensors such as Inertial measurement Unit has made it possible for other information of the human hand activities 
TABLE IV: Strengths and weaknesses of the pHRC sensors

\begin{tabular}{lll}
\hline Sensing method & Strengths & Weaknesses \\
\hline Vision-based sensors & $\begin{array}{l}\text { Popular, available support } \\
\text { from community of users, } \\
\text { easy to use. }\end{array}$ & $\begin{array}{l}\text { Affected by occlusion, } \\
\text { suffers from light } \\
\text { conditions such as } \\
\text { reflection and contrast. }\end{array}$ \\
\hline $\begin{array}{l}\text { Optical-based } \\
\text { tactile robotic } \\
\text { skin sensor }\end{array}$ & $\begin{array}{l}\text { Grasp stability and } \\
\text { slip detection, contact } \\
\text { force and torque estimation, } \\
\text { robust to electrical } \\
\text { interference. }\end{array}$ & $\begin{array}{l}\text { Fragility and rough } \\
\text { estimation. }\end{array}$ \\
\hline $\begin{array}{l}\text { Soft tactile } \\
\text { robotic skin } \\
\text { sensor }\end{array}$ & $\begin{array}{l}\text { Suitable for object } \\
\text { exploration, contact point } \\
\text { manipulation, object } \\
\text { recognition, texture \& } \\
\text { classification. }\end{array}$ & $\begin{array}{l}\text { Ageing and mechanical } \\
\text { Stress causes hysteresis \& } \\
\text { reduction in the sensitivity } \\
\text { of the sensor. }\end{array}$ \\
\hline & Versatile and quick & $\begin{array}{l}\text { Drift in sensing output, } \\
\text { information redundancy, } \\
\text { required advance processing } \\
\text { technique to extract reliable } \\
\text { control signal. }\end{array}$ \\
EMG/EEG & Complementary inputs. & $\begin{array}{l}\text { calibration required, selection } \\
\text { is technically specific, limited } \\
\text { portability. }\end{array}$ \\
\hline Data Glove & &
\end{tabular}

such as acceleration, angular velocity, and magnetic field to be measured using the data glove [82], [83]. Fang et al. proposed a novel data glove which can capture human arm-hand motions simultaneously [84]. This allows the glove to be fully mapped to a robotic arm-hand system, hence allowing the robot to perform intuitive motions when teleoperated. For convenience sake, the device is compact, portable and uses Bluetooth for operational data transfer to the robotic system.

\section{B. Actuators for Collaborative Robots}

Generally, actuators could be categorised into pneumatic which uses compressed air to generate motion, hydraulic which uses compressed fluid to generate motion, electric which uses electric current or magnet to generate motion, and a hybrid of the aforementioned categories.

1) Pneumatic Technologies: Pneumatic technology has been applied to create artificial muscle in robot hands [85], [86] and to generate motion in soft-robotics [87], [88]. This kind of actuator is desirable in safety-conscious robotic design especially those intended to interact with human and delicate contact surfaces [87]. One of its drawbacks is that it is difficult to control due to the non-linearity of the actuator. Considering the unpredictable and unstructured nature of the space environment, Booth et al. proposed a multi-functional robotic skin to enable complex motions and functions [89].

The device integrates both actuation and sensing into a single material to enable multiple motions control that can turn inanimate objects into multifunctional robots. The skin is modulated (see Fig. 11a) and could be wrapped around deformable objects (see Fig. 11b) so they could be manipulated (see Fig. 11c) to achieve complex motions (see Fig. 11d). The devices are easy to be removed and applied on other objects with little or no reconfiguration.

2) Electric Motors: Electric motors often produce high speed but poor torque density. By using reduced gear technique, the torque could be improved but with a trade-off to speed. Other disadvantages of using reduced gear technique include that it introduces friction, backlash, torque ripple and noise to the robotic systems [90]. Because of these challenges,

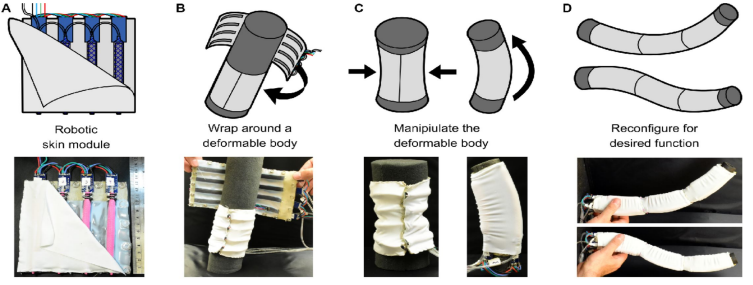

Fig. 11: (a) The robotic skins with embedded distributed actuators used to transform inanimate object into a moving object. (b) The robotic skins can be wrapped around deformable objects to produce different forms of deformetions as shown in (c). (d) Multiple robotic skins can be wrapped on a deformable object to produce complex motions [89].

TABLE V: Strengths and weaknesses of the pHRC actuators

\begin{tabular}{lll}
\hline Sensing method & Strengths & Weaknesses \\
\hline Pneumatic Technologies & $\begin{array}{l}\text { Desirable in safety-conscious } \\
\text { robotic design, especially those } \\
\text { intended to interact with } \\
\text { human and delicate contact surfaces. }\end{array}$ & $\begin{array}{l}\text { Difficult to control due to } \\
\text { the non-linearity of the } \\
\text { actuator. }\end{array}$ \\
\hline Electric Motors & $\begin{array}{l}\text { Has good shock absorbing } \\
\text { properties, } \\
\text { robust to electrical } \\
\text { interference. }\end{array}$ & $\begin{array}{l}\text { Often produces poor } \\
\text { torque density. }\end{array}$ \\
\hline Hydraulic Actuators & $\begin{array}{l}\text { Can provide high torque, } \\
\text { stays robust to burnout, } \\
\text { and can maintain very strong } \\
\text { linear motion without gears. }\end{array}$ & $\begin{array}{l}\text { Susceptible to the temperature } \\
\text { of the liquid it is made of \& } \\
\text { difficult to miniaturize. }\end{array}$ \\
\hline
\end{tabular}

the Series Elastic Actuator (SEA) could be used in their place. The SEA protects the robot from shock when it collides with an object [57]. It is even applied in exoskeletons for interactive force control between human and the robot coworker [91]. Despite the success of SEA, it may not be the best actuator for robots that need high degrees of stiffness. In that case, Variable Stiffness Actuator (VSA) is the remedy [92], [93]. The technology could also be in the form of Variable Impedance Actuator (VIA) which varies its stiffness as a response to change in the impedance [94]. VSA and VIA actuators have a better shock absorbing properties than SEA as they can store and release energy in passive elastic elements [95].

3) Hydraulic Actuators: The hydraulic actuator is good for carrying heavy loads and it is generally known to have fewer problems when exposed to heat. It can also provide high torque, stays robust to burnout and maintain very strong linear motion without gears. However, hydraulic actuators could be susceptible to the temperature of the liquid used and difficult to miniaturize. Despite, its limitations, it has found favour in wearable and in mobile robotic systems as demonstrated in [96] and in [97] where the authors utilized the actuator to assist human users to carry a heavier load.

\section{Collaborative StRategies of PHRC}

Collaborative strategies deal with different levels of ideas: joint attention formulation, turn-taking, task planning and knowledge representation.

\section{A. Joint Attention Formulation}

Several techniques and methods have been used in different fields to bring the attention of individual towards activating 
their readiness to participate in a joint task or to establish either a common ground or bond between the minds of the collaborators [98], [99]. A successfully formulated joint attention draws the participants towards a shared focus on an object or other areas of interest [99]. These techniques have been successfully applied in HRI for children with Autism Spectrum Disorders (ASD) [100], [101]. Attention formulation could be achieved either by deictic words or vocal expressions such as "look here, see me, look right, or next one”, by pointing gesture, by using line of sight, by using communication cue such as eye gazing [102], [103] or by combination of vocal and gesture commands [104], [105].

\section{B. Turn-Taking}

Turn-taking is one of the mechanisms for coordinating smooth and excellent conversation between two or more persons [106]. This idea has advanced from human-human conversation to earn applications in human-robot interaction and collaboration [107]. Several approaches have been proposed to implement turn-taking. Among others are the use of signaling approach such as gaze [108], [109], gesture [110] and body language [111]; and by means of verbal communications [107]. Not only does a robot needs to understand when turntaking occurs, but it is also necessary for a robot to recognize when a human is yielding to a turn and the end of a turn for a timely response to be given to the human partner.

\section{Task Planning and Knowledge Representation}

The most commonly used task planner is the Human Aware Task Planner (HATP). HATP is a hierarchical task planning approach that is capable of taking into account both the state and the task preferences of a human in a collaborative task with a robot as demonstrated in [112], [113]. Similarly, a Human Aware Task Motion Planning was presented in [114], to endow a robot with the ability to estimate human intents and produce acceptable behaviours. Furthermore, some researchers have also employed Stanford Research Institute Problem Solver (STRIP) planner due to its simplicity and capability to solidly represent a domain of interest [115], [116]. Continues update of symbolic knowledge about the robot belief of the world could be made available and maintained in a knowledge base like the Spatial Reasoning Knowledge (SPARK) [117]. Sisbot et al. in [118] proposed a situation assessment framework that focused on HRC in the object manipulation. The authors built an Open Robots Ontology (ORO) knowledge-base to maintain a symbolic representation of the state of the world.

\section{Robot Learning Methodologies}

It is practically impossible to precode a robot to cope with all the actions needed to satisfactorily collaborate with humans in a complex and dynamic environment like ours. Because of this reason, learning algorithms are employed to facilitate the ability of a robotic system to cope in such situations.

Recently, Artificial Neural Network (ANN) and its derivative Deep Learning are increasingly applied in different scenarios involving pHRC. A neural network human emotional expression recognition model was proposed in [119]. In the paper, Vircikova et al. trained a humanoid robot to learn a human emotional expression and respond to it accordingly. Also in [120], the authors applied Recurrent Neural Networks (RNNs) to learn surgical knotting for Minimally Invasive Surgery framework. Yang et al. [121] proposed a Neurallearning-based telerobot control at both kinematic and dynamic levels. Neural networks are generally known for having strong approximation and good at handling redundancy, noise control and a large volume of data [122]. Pinto and Gupta in [123], used a Convolutional Neural Networks to train a robot to randomly grasp an object. The authors argued that their method eliminates the issue of bias that often come up when a human being manually annotates grasping location of the objects. The two major drawbacks of Deep Learning are that it requires a long training period and has high computational cost [124].

Support Vector Machine (SVM) is another technique that has earned popularity in human action recognition and human motion prediction due to its good recognition accuracy and fast training time [125]. In [126], the authors proposed a model to optimize task performance human-robot collaborative minimally invasive surgery operations using SVM classifier. The SVM classifier was used to train the robot to predict the future human motion [127] which enables it to estimate human's region of interest and comply accordingly.

Gaussian Mixture Model (GMM) is one of the most popular methods amongst the learning techniques employed in pHRC research work. Some of the features that made it unique are:

1) The ability to tolerate an arbitrarily large number of Gaussian components and a small number of variances.

2) The fast convergence process, which makes it computational inexpensive.

GMM technique was systematically used in [128] to perform a human to a robot object manipulation skill transfer based on active learning. In the work, the robot observed the demonstration via motion sensing system while GMM was used to encode sets of trajectories gathered from the sensor. Furthermore, GMM was employed in [129] to learn a trajectory tube insertion for surgery task. Rozo et al. [130] proposed a framework to exploit both position and force data in HRC. The authors tested the proposed technique on two different experiments involving robot handling both position and force constraints in a collaborative box transportation task and collaborative assembly of a wooden IKEA table.

Hidden Markov Model (HMM) has been widely used to model robot learning collaborative interaction with humans [131]-[133]. HMM is derived from a Markov process which believes that the current state of a system depends solely on the system's prior state. Several authors have suggested using HMM to encode and reproduce demonstrated action where noise in the observation is inevitable [134], [135].

Dynamic Movement Primitive (DMP) has been extensively used in pHRC especially in learning control policy of human motion trajectories [136], [137]. Prada et al. [138] proposed a generic DMP framework for ensuring human-friendly and fluent robot motion in object hand-over interaction between human and robot collaborator. Using a collection of sequences 
of captured motion configurations of two people demonstrating object hand-over task in an industrial context, the authors trained a robot to learn the control motion hand-over task.

Reinforcement Learning (RL) applies a reward policy upon which an agent depends to improve its learned behaviour. In $\mathrm{RL}$, the error outcome of a previously performed action is used to update the action state policy of the robot thereby serving as a way of strengthening the belief state of the robot [139]. In [140], the authors proposed a RL technique to improve the rate at which a robot learns dynamic tasks and motor primitives [141]. In this case, the tasks learned are to hold a table in a suitable position and cooperatively lift the table with human collaborator while keeping it horizontal. Dimeas et al. in [142] applied reinforcement learning technique to learn variable admittance control for human-robot co-manipulation.

\section{Safety Issues, Control Designs and Human FACTORS}

\section{A. Safety Issues}

The coexistence of human and robot poses a safety threat both to humans and the collaborating environment. Because of that, the ISO enacted a safety standard (ISO TS 15066) for the collaborative industrial robotic systems as specified in [9]. Hence most of the recently produced collaborative robots have the collision detection sensors embedded in them. The sensors could be in the form of an embedded accelerometer, tactile sensors or current feedback which could be felt when an abnormal force is sensed by the robot [143]. Other approaches applied in safety include collision avoidance and collision detection. This feature could be achieved in robot applications by proper motion planning and control of some sensory systems [144], [145]. In [146], the authors used a potential field approach to implement collision avoidance. In their work, repulsive and attractive fields were associated with the obstacle and the target objects respectively.

\section{B. Control Designs in pHRC}

In the context of this research, pHRC control is classified into direct, supervisory and fully collaborative controls as presented in Figure 12.

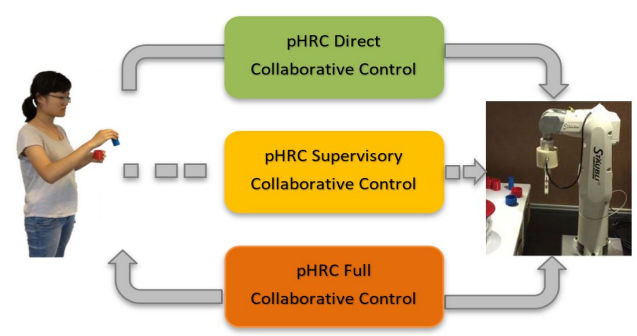

Fig. 12: A block diagram to describe the different design control concepts in pHRC.

In direct control design, a human is totally in control of the robot manipulation and as such the control decision is wholly taken by a human while the robotic system plays a passive role only. This is not an efficient system control design as the performance of the operation depends soley on the skills and experience of the human operator. This kind of control model has found applications in motor control learning [147]-[149] and robot imitating human actions [150]. Using agonistic and antagonistic signals extracted from the human joint, Zhijun et al. [151] proposed EMG based upper-limb robot assistant exoskeleton control system. In the paper, the authors employed linear discriminant analysis-based classifier to indicate the kind of motion in the joint which enables them to estimate the torque control signal.

In supervisory control design, human and robot establish a shared control or traded control scenario. A robot makes most of the decision while humans are required in the control loop occasionally and when necessary. This contributes so much in reducing the idle time of a robotic system in operation. A group of researchers from NASA's Johnson Space Center (JSC) has developed a graphic interface suite called Predictive Interactive Graphical Interface (PIGI) to accomplish suspensory control of a robot in the space using a communication latency of 5-10 sec. [152].

In fully collaborative control, the robot is made to be aware of its environment and play both adaptive and selfreliance roles hence, making the collaboration between human and robot intuitive. A lot of efforts have been made to address the interactive behaviour between human and robot as demonstrated in [153]. In [154], the authors proposed a robot adaptive control focusing on allowing the robot to play the roles of either a task leader or follower based on the intention of human collaborator. Using game theory, Li et al. [155] proposed a role adaptive framework that enables the robot to adjust its role in accordance with human intention.

\section{Human Factors}

Several human factors could be issues in the usability, effectiveness, safety, and administering of a robotic system for human-robot collaboration. Based on this, administering exoskeleton to a subject could be challenging due to the vast variation and compositions of various human body parts. In Cybathlon [53], earlier evaluation revealed that the bone mineral density of the subject was within the normal limit, however, later evaluation showed that the rate of motion of his knee joints has been limited due to prolonged use of a wheelchair. Hence, how to analyze wearable robotic devices and prosthesis to obtain the best physical form remains a challenge. Human attitude and perception toward robotic systems are essential in the performance cooperation of humanrobot teammate. The level of trust will directly affect the willingness of a human teammate to follow robot's suggestions or instructions [156]. The need for the robotic system and a human team worker to build a mental model of each other has been suggested long ago as it would reduce the risk of human error and ensure safer interaction of them [157], [158].

\section{DISCUSSION AND CONCLUSION}

Considering the unstructured and dynamic nature of the human environment, most of the collaborative robots are 
equipped with inbuilt sophisticated sensors, collaborative interfaces and enhanced control systems to improve safety and collaborative abilities of the robotic systems. Furthermore, the enhanced physical dexterity of the robotic systems has paved the way for their potential application and relevance in various aspects of human endeavours. Similarly, wearable and assistive robotic systems have withnessed rapid development in the last decades. This has led to the development of improved systems that could allow human upper or lower limbs movements in multiple dimensions. Despite the advancement in this research area, it is still saddled with the challenges of accomplishing a compact, lightweight and comfortably aligned upper and lower limp exoskeletons [159]. Hence, limiting the ability of the systems to be employed in accomplishing complex interaction with human users. Furthermore, research in this area is still in a premature state as most of the proposed systems are just prototypes and have not been evaluated across different application scenarios.

Recent trends in EEG technology is looking at extracting human neural response with different spatial frequencies for estimating human intention [160]. Future research direction could look at developing a high-density EEG system that can capture more neural information for better inference of the brain activities. Different kinds of sensors are currently employed in the design of data glove to provide high accuracy and to measure the activities of human fingers. Apart from designing low-cost data gloves, currently, researchers have focused on modular and expansible data glove design in order to improve adaptability [82]. Future research direction includes the addition of multiple feedback in a single finger of the data glove while ensuring consistent data across a large range of the hand. Some researchers have coupled more than one sensing devices together in order to produce more capable and adaptive sensors such as vision-based tactile sensors. The prospect of this system in the future is promising as it possesses the ability to enable the robotic hand to process complex dexterous manipulative tasks. Moreover, future research direction could focus on using new flexible materials and innovative mechanisms to further achieve enhanced soft skin-based sensors suitable for practical applications in the field of medical implant services, and in other potential fields.

A lot of learning systems have been proposed to equip a robot with cognitive and cooperative capabilities that will enable it to understand its environment, acquire desired skills that will enhance its collaborative abilities with humans but there are still some challenges seeking for solutions. For instance, it is still challenging for a robot to infer the state and belief of a human collaborator with multi-directional intents. It is also very hard to model and transfer human preference because of the variation in peoples' preferences. With this little knowledge variance, research on robot predicting human preferences is still an open research question. In addition, Deep Learning technique has been applied in several applications including in robotic perception for object detection, object recognition, robotic grasps identification, environmental and place recognition and, for learning sensory-motor control. However, few previous studies have investigated applying Deep Learning-based methods in pHRC; thus, further studies are needed.

In pHRC, safety collaboration is of paramount importance and still have several unanswered research questions. To the best of our knowledge, no research work has fully answered the question "what is the best way to differentiate between accidental collision and simply touch from a robot to a human?" Improving ergonomically collision detection and avoidance in pHRC is seeking further research attention.

Finally, an overview of the state-of-the-art Physical HumanRobot Collaboration ( $\mathrm{pHRC}$ ) cutting across the hardware and software concerning the implementation of effective collaboration has been reviewed. Considering the wide coverage and rigorous studies carried out in this survey, the authors have no doubt that the outcome could serve as a guide or starting point to scholars interested in $\mathrm{pHRC}$.

\section{ACKNOWLEDGMENT}

The authors would like to thank all the members of the Intelligent Systems \& Biomedical Robotics Group (ISR) at the University of Portsmouth for the wonderful feedbacks on the content and scope of this article.

\section{REFERENCES}

[1] S. Yang, X. Mao, Z. Liu, S. Yang, J. Xue, and Z. Xu, "The accompanying behavior model and implementation architecture of autonomous robot software," in 2017 24th Asia-Pacific Software Engineering Conference (APSEC). IEEE, 2017, pp. 209-218.

[2] T. Munzer, M. Toussaint, and M. Lopes, "Preference learning on the execution of collaborative human-robot tasks," in 2017 IEEE International Conference on Robotics and Automation (ICRA), May 2017, pp. 879-885.

[3] S. Grahn, B. Langbeck, K. Johansen, and B. Backman, "Potential advantages using large anthropomorphic robots in human-robot collaborative, hand guided assembly," Procedia CIRP, vol. 44, pp. 281-286, 2016.

[4] P. Salvini, M. Nicolescu, and H. Ishiguro, "Benefits of human-robot interaction," IEEE Robotics \& Automation Magazine, vol. 18, no. 4, pp. 98-99, 2011.

[5] B. Huang, Z. Li, X. Wu, A. Ajoudani, A. Bicchi, and J. Liu, "Coordination control of a dual-arm exoskeleton robot using human impedance transfer skills," IEEE Transactions on Systems, Man, and Cybernetics: Systems, pp. 1-10, 2018.

[6] Z. Li, C. Xu, Q. Wei, C. Shi, and C. Su, "Human-inspired control of dual-arm exoskeleton robots with force and impedance adaptation," IEEE Transactions on Systems, Man, and Cybernetics: Systems, pp. $1-10,2018$.

[7] "Robots and robotic devices - safety requirements for industrial robots - part 1: Robots." https://www.iso.org/standard/51330.html, 2011, accessed: 2017-08-14.

[8] "Robots and robotic devices - safety requirements for industrial robots - part 1: Robots.” https://www.iso.org/standard/41571.html, 2011, accessed: 2017-08-14.

[9] "Robots and robotic devices - collaborative robots." https://www.iso.org/standard/62996.html, 2016, accessed: 2018-07-04.

[10] K. Hernandez, B. Bacca, and B. Posso, "Multi-goal path planning autonomous system for picking up and delivery tasks in mobile robotics," IEEE Latin America Transactions, vol. 15, no. 2, pp. 232 238, 2017.

[11] M. A. Goodrich, A. C. Schultz et al., "Human-robot interaction: a survey," Foundations and Trends ${ }^{\circledR}$ in Human-Computer Interaction, vol. 1, no. 3, pp. 203-275, 2008.

[12] B. Chandrasekaran and J. M. Conrad, "Human-robot collaboration: A survey," in SoutheastCon 2015. IEEE, 2015, pp. 1-8.

[13] V. Villani, F. Pini, F. Leali, and C. Secchi, "Survey on humanrobot collaboration in industrial settings: Safety, intuitive interfaces and applications," Mechatronics, 2018.

[14] A. Bicchi, M. A. Peshkin, and J. E. Colgate, "Safety for physical human-robot interaction," in Springer handbook of robotics. Springer, 2008, pp. 1335-1348. 
[15] A. Ajoudani, A. M. Zanchettin, S. Ivaldi, A. Albu-Schäffer, K. Kosuge, and O. Khatib, "Progress and prospects of the human-robot collaboration," Autonomous Robots, pp. 1-19, 2018.

[16] "CNN Tech-Rethink Robotics." http://money.cnn.com/2015/04/07/ technology/sawyer-robot-manufacturing-revolution/, accessed: 2010$10-23$.

[17] "Universal Robotics, Find the right 6 axis robot." https://www.universal-robots.com/products/help-me-choose/, accessed: 2010-10-23.

[18] "APAS ASSISTANT, The new era of robotic co-workers." http://essert.com/collaborating-robots/?lang=en, accessed: 2010-10-23.

[19] "Collaborative robot cr-35ia." http://www.fanuc.eu/de/en/robots/robotfilter-page/collaborative-robots/collaborative-cr35ia, accessed: 201711-31.

[20] "ABB Robotics, The new era of robotic co-workers." http://new.abb.com/products/robotics/industrial-robots/yumi, accessed: 2010-10-22.

[21] "Next generation industrial robot, available a:," http://nextage.kawada.jp/en/specification/, accessed: 2016-12-31.

[22] "Pr2 manual:", https://pr2s.clearpathrobotics.com/wiki/PR220Manual, accessed: 2016-12-31.

[23] "IRB 14000 YuMi - Industrial Robots - Robotics abb." http://new.abb.com/products/robotics/industrial-robots/yumi, accessed: 2010-10-29.

[24] "Hardware and software platform for mobile manipulation research and development," http://www.willowgarage.com/pages/pr2/design, accessed: 2016-12-31.

[25] S. Cousins, "Ros on the pr2 [ros topics]," IEEE Robotics \& Automation Magazine, vol. 17, no. 3, pp. 23-25, 2010.

[26] A. Van Delden, C. L. E. Peper, G. Kwakkel, and P. J. Beek, "A systematic review of bilateral upper limb training devices for poststroke rehabilitation," Stroke research and treatment, vol. 2012, 2012.

[27] A. Otten, C. Voort, A. Stienen, R. Aarts, E. van Asseldonk, and H. van der Kooij, "Limpact: A hydraulically powered self-aligning upper limb exoskel-eton," IEEE/ASME transactions on mechatronics, vol. 20, no. 5, pp. 2285-2298, 2015.

[28] F. Just, K. Baur, R. Riener, V. Klamroth-Marganska, and G. Rauter, "Online adaptive compensation of the armin rehabilitation robot," in Biomedical Robotics and Biomechatronics (BioRob), 2016 6th IEEE International Conference on. IEEE, 2016, pp. 747-752.

[29] H.-C. Hsieh, D.-F. Chen, L. Chien, and C.-C. Lan, "Design of a parallel actuated exoskeleton for adaptive and safe robotic shoulder rehabilitation," IEEE/ASME Transactions on Mechatronics, vol. 22, no. 5, pp. 2034-2045, 2017.

[30] "Wearable robotics exoskeletons, bionics lab." http://bionics.seas.ucla.edu/, accessed: 2017-09-11.

[31] Z. Li, B. Huang, A. Ajoudani, C. Yang, C. Su, and A. Bicchi, "Asymmetric bimanual control of dual-arm exoskeletons for humancooperative manipulations," IEEE Transactions on Robotics, vol. 34, no. 1, pp. 264-271, Feb 2018.

[32] M. Mihelj, T. Nef, and R. Riener, "Armin ii-7 dof rehabilitation robot: mechanics and kinematics," in Robotics and Automation, 2007 IEEE International Conference on. IEEE, 2007, pp. 4120-4125.

[33] Z. Li, B. Huang, Z. Ye, M. Deng, and C. Yang, "Physical humanrobot interaction of a robotic exoskeleton by admittance control," IEEE Transactions on Industrial Electronics, vol. 65, no. 12, pp. 9614-9624, Dec 2018.

[34] P. Maciejasz, J. Eschweiler, K. Gerlach-Hahn, A. Jansen-Troy, and S. Leonhardt, "A survey on robotic devices for upper limb rehabilitation," Journal of neuroengineering and rehabilitation, vol. 11, no. 1, p. 3, 2014.

[35] H. Aguilar-Sierra, W. Yu, S. Salazar, and R. Lopez, "Design and control of hybrid actuation lower limb exoskeleton," Advances in Mechanical Engineering, vol. 7, no. 6, p. 1687814015590988, 2015.

[36] Y. Bai, F. Li, J. Zhao, J. Li, F. Jin, and X. Gao, "A powered anklefoot orthoses for ankle rehabilitation," in 2012 IEEE International Conference on Automation and Logistics, Aug 2012, pp. 288-293.

[37] K. A. Shorter, G. F. Kogler, E. Loth, W. K. Durfee, and E. T. HsiaoWecksler, "A portable powered ankle-foot orthosis for rehabilitation." Journal of Rehabilitation Research \& Development, vol. 48, no. 4, 2011.

[38] R. Jimenez-Fabian and O. Verlinden, "Review of control algorithms for robotic ankle systems in lower-limb orthoses, prostheses, and exoskeletons," Medical engineering \& physics, vol. 34, no. 4, pp. $397-$ $408,2012$.
[39] S. H. Collins, M. B. Wiggin, and G. S. Sawicki, "Reducing the energy cost of human walking using an unpowered exoskeleton," Nature, vol. 522, no. 7555 , p. $212,2015$.

[40] L. M. Mooney, E. J. Rouse, and H. M. Herr, "Autonomous exoskeleton reduces metabolic cost of human walking," Journal of neuroengineering and rehabilitation, vol. 11, no. 1, p. 151, 2014.

[41] Z. Li, C. Deng, and K. Zhao, "Human cooperative control of a wearable walking exoskeleton for enhancing climbing stair activities," IEEE Transactions on Industrial Electronics, pp. 1-1, 2019.

[42] K. Li, Y. Fang, Y. Zhou, and H. Liu, "Non-invasive stimulationbased tactile sensation for upper-extremity prosthesis: a review," IEEE Sensors Journal, vol. 17, no. 9, pp. 2625-2635, 2017.

[43] U. Wijk and I. Carlsson, "Forearm amputees' views of prosthesis use and sensory feedback," Journal of Hand Therapy, vol. 28, no. 3, pp. 269-278, 2015.

[44] A. Mingrino, A. Bucci, R. Magni, and P. Dario, "Slippage control in hand prostheses by sensing grasping forces and sliding motion," in Intelligent Robots and Systems' 94.'Advanced Robotic Systems and the Real World', IROS'94. Proceedings of the IEEE/RSJ/GI International Conference on, vol. 3. IEEE, 1994, pp. 1803-1809.

[45] D. A. Osborn, H. Betthauser, K. Nguyen, and T. V, "Prosthesis with neuromorphic multilayered e-dermis perceives touch and pain," Science, vol. 3, no. 3, 2018.

[46] R. Masuda, K. Hasegawa, and K. Osako, "Slip sensor for industrial robot and its application," The transactions of the Institute of Electrical Engineers of Japan. C, vol. 96, no. 10, pp. 219-226, 1976.

[47] Y. Yamada, K. Sanda, K. Fujita, N. Tsuchida, and K. Imai, "Active sensing of static friction coefficient $\mu$ for controlling grasping force," Transactions of the Society of Instrument and Control Engineers, vol. 30, no. 10, pp. 1188-1194, 1994.

[48] B. E. Lawson, J. Mitchell, D. Truex, A. Shultz, E. Ledoux, and M. Goldfarb, "A robotic leg prosthesis: Design, control, and implementation," IEEE Robotics \& Automation Magazine, vol. 21, no. 4, pp. 70-81, 2014.

[49] T. D. Barnett, "Interesting engineering,." https://interestingengineering.com/this-robotic-arm-named-after-lukeskywalker-is-pushing-the-boundaries-of-prosthetics, 2017, accessed: 2019-04-1.

[50] "Assist robots for industrial use." https://news.panasonic.com/2016/44969.html, 2016, accessed: 201807-07.

[51] "Assist robots for industrial use." https://exoskeletonreport.com/, 2016, accessed: 2018-07-07.

[52] Y. Ikeuchi, J. Ashihara, Y. Hiki, H. Kudoh, and T. Noda, "Walking assist device with bodyweight support system," in Intelligent Robots and Systems, 2009. IROS 2009. IEEE/RSJ International Conference on. IEEE, 2009, pp. 4073-4079.

[53] J. Choi, B. Na, P.-G. Jung, D.-w. Rha, and K. Kong, "Walkon suit: A medalist in the powered exoskeleton race of cybathlon 2016," IEEE Robotics \& Automation Magazine, vol. 24, no. 4, pp. 75-86, 2017.

[54] J. E. Colgate, M. Peshkin, and S. H. Klostermeyer, "Intelligent assist devices in industrial applications: a review," in Intelligent Robots and Systems, 2003.(IROS 2003). Proceedings. 2003 IEEE/RSJ International Conference on, vol. 3. IEEE, 2003, pp. 2516-2521.

[55] A. Campeau-Lecours, S. Foucault, T. Laliberté, B. Mayer-St-Onge, and C. Gosselin, "A cable-suspended intelligent crane assist device for the intuitive manipulation of large payloads," 2016.

[56] D. Zhang, R. Ikeura, and Y. Mori, "Motion reproduction by human demonstration based on discrete hid-den markov model for nursing-care assistant robot," in 2014 IEEE International Conference on Systems. Man, and Cyber-netics (SMC). IEEE, 2014, pp. 842-846.

[57] H. Reddivari, C. Yang, Z. Ju, P. Liang, Z. Li, and B. Xu, "Teleoperation control of baxter robot using body motion tracking," in Multisensor Fusion and Information Integration for Intelligent Systems (MFI), 2014 International Conference on. IEEE, 2014, pp. 1-6.

[58] Y. Xu, C. Yang, J. Zhong, N. Wang, and L. Zhao, "Robot teaching by teleoperation based on visual interaction and extreme learning machine," Neurocomputing, vol. 275, pp. 2093 - 2103, 2018.

[59] G. Konidaris, S. Kuindersma, R. Grupen, and A. Barto, "Robot learning from demonstration by constructing skill trees," The International Journal of Robotics Research, vol. 31, no. 3, pp. 360-375, 2012.

[60] E. A. Billing and T. Hellström, "A formalism for learning from demonstration," Paladyn, Journal of Behavioral Robotics, vol. 1, no. 1, pp. 1-13, 2010.

[61] K. Li, Y. Fang, Y. Zhou, and H. Liu, "Non-invasive stimulationbased tactile sensation for upper-extremity prosthesis: A review," IEEE Sensors Journal, vol. 17, no. 9, pp. 2625-2635, May 2017. 
[62] R. S. Dahiya, P. Mittendorfer, M. Valle, G. Cheng, and V. J. Lumelsky, "Directions toward effective utilization of tactile skin: A review," IEEE Sensors Journal, vol. 13, no. 11, pp. 4121-4138, Nov 2013.

[63] A. Yamaguchi and C. G. Atkeson, "Combining finger vision and optical tactile sensing: Reducing and handling errors while cutting vegetables," in 2016 IEEE-RAS 16th International Conference on Humanoid Robots (Humanoids), Nov 2016, pp. 1045-1051.

[64] A. Yamaguchi and C. G. Atkeson, "Implementing tactile behaviors using fingervision," in 2017 IEEE-RAS 17th International Conference on Humanoid Robotics (Humanoids). IEEE, 2017, pp. 241-248.

[65] P. Maiolino, M. Maggiali, G. Cannata, G. Metta, and L. Natale, "A flexible and robust large scale capacitive tactile system for robots," IEEE Sensors Journal, vol. 13, no. 10, pp. 3910-3917, Oct 2013.

[66] B. D. Argall and A. G. Billard, "A survey of tactile human-robot interactions," Robotics and autonomous systems, vol. 58, no. 10, pp. 1159-1176, 2010.

[67] T. Kawasetsu, T. Horii, H. Ishihara, and M. Asada, "Mexican-hatlike response in a flexible tactile sensor using a magnetorheological elastomer," Sensors, vol. 18, no. 2, p. 587, 2018

[68] M. Kaboli and G. Cheng, "Novel tactile descriptors and a tactile transfer learning technique for active in-hand object recognition via texture properties," in IEE-RAS International Conference on Humanoid Robots-Workshop Tactile sensing for manipulation: new progress and challenges, 2016.

[69] M. Kaboli and G. Cheng, "Robust tactile descriptors for discriminating objects from textural properties via artificial robotic skin," IEEE Transactions on Robotics, vol. 34, no. 4, pp. 985-1003, Aug 2018.

[70] H. Liu, "Exploring human hand capabilities into embedded multifingered ob-ject manipulation," IEEE Transactions on Industrial Informatics, vol. 7, no. 3, pp. 389-398, 2011.

[71] K. Gui, H. Liu, and D. Zhang, "Toward multimodal humanrobot interaction to enhance active participation of users in gait rehabilitation," IEEE Transactions on Neural Systems and Rehabilitation Engineering, vol. 25, no. 11, pp. 2054-2066, Nov 2017.

[72] C. Yang, C. Zeng, P. Liang, Z. Li, R. Li, and C. Y. Su, "Interface design of a physical human-robot interaction system for human impedance adaptive skill transfer," IEEE Transactions on Automation Science and Engineering, vol. PP, no. 99, pp. 1-12, 2017.

[73] Y. Mangukiya, B. Purohit, and K. George, "Electromyography (emg) sensor controlled assistive orthotic robotic arm for forearm movement," in Sensors Applications Symposium (SAS), 2017 IEEE. IEEE, 2017, pp. $1-4$.

[74] E. M. Faidallah, Y. H. Hossameldin, S. M. A. Rabbo, and Y. A. ElMashad, "Control and modeling a robot arm via emg and flex signals," in 15th International Workshop on Research and Education in Mechatronics (REM), 2014.

[75] C. Yang, P. Liang, A. Ajoudani, Z. Li, and A. Bicchi, "Development of a robotic teaching interface for human to human skill transfer," in Intelligent Robots and Systems (IROS), 2016 IEEE/RSJ Interna-tional Conference on. IEEE, 2016, pp. 710-716.

[76] A. T. Tzallas, N. Giannakeas, K. N. Zoulis, M. G. Tsipouras, E. Glavas, K. D. Tzimourta, L. G. Astrakas, and S. Konitsiotis, "Eeg classification and short-term epilepsy prognosis using brain computer interface software," in 2017 IEEE 30th International Symposium on ComputerBased Medical Systems (CBMS), June 2017, pp. 349-353.

[77] J. Gutierrez-Cáceres, C. Portugal-Zambrano, and C. Beltran-Castanon, "Computer aided medical diagnosis tool to detect normal/abnormal studies in digital $\mathrm{mr}$ brain images," in Computer-Based Medical Systems (CBMS), 2014 IEEE 27th International Symposium on. IEEE, 2014, pp. 501-502.

[78] M. Y. Latif, L. Naeem, T. Hafeez, A. Raheel, S. M. U. Saeed, M. Awais, M. Alnowami, and S. M. Anwar, "Brain computer interface based robotic arm control," in 2017 International Smart Cities Conference (ISC2), Sept 2017, pp. 1-5.

[79] C. Yang, H. Wu, Z. Li, W. He, N. Wang, and C. Y. Su, "Mind control of a robotic arm with visual fusion technology," IEEE Transactions on Industrial Informatics, vol. PP, no. 99, pp. 1-1, 2017.

[80] D. J. Sturman and D. Zeltzer, "A survey of glove-based input," IEEE Computer graphics and Applications, vol. 14, no. 1, pp. 30-39, 1994.

[81] C.-P. Tung and A. C. Kak, "Automatic learning of assembly tasks using a dataglove system," in Intelligent Robots and Systems 95.'Human Robot Interaction and Cooperative Robots', Proceedings. 1995 IEEE/RSJ International Conference on, vol. 1. IEEE, 1995, pp. 1-8.

[82] B.-S. Lin, I. Lee, S.-Y. Yang, Y.-C. Lo, J. Lee, J.-L. Chen et al., "Design of an inertial-sensor-based data glove for hand function evaluation," Sensors, vol. 18, no. 5, p. 1545, 2018.
[83] K. Kitano, A. Ito, N. Tsujiuchi, and S. Wakida, "Estimation of joint center and measurement of finger motion by inertial sensors," in 2016 38th Annual International Conference of the IEEE Engineering in Medicine and Biology Society (EMBC), Aug 2016, pp. 5668-5671.

[84] B. Fang, D. Guo, F. Sun, H. Liu, and Y. Wu, "A robotic hand-arm teleoperation system using human arm/hand with a novel data glove," in 2015 IEEE International Conference on Robotics and Biomimetics (ROBIO), Dec 2015, pp. 2483-2488.

[85] J. Yi, Z. Shen, C. Song, and Z. Wang, "A soft robotic glove for hand motion assistance," in 2016 IEEE International Conference on Realtime Computing and Robotics (RCAR), June 2016, pp. 111-116.

[86] R. Kang, Y. Guo, K. Cheng, and L. Chen, "Design and control of a soft actuator driven by pneumatic muscles," in 2014 International Conference on Industrial Automation, Information and Communications Technology, Aug 2014, pp. 26-30.

[87] C. J. Payne, I. Wamala, C. Abah, T. Thalhofer, M. Saeed, D. BautistaSalinas, M. A. Horvath, N. V. Vasilyev, E. T. Roche, F. A. Pigula et al., "An implantable extracardiac soft robotic device for the failing heart: Mechanical coupling and synchronization," Soft Robotics, 2017.

[88] D. Baiden and O. Ivlev, "Human-robot-interaction control for orthoses with pneumatic soft-actuatorsconcept and initial trails," in Rehabilitation robotics (ICORR), 2013 IEEE international con-ference on. IEEE, 2013, pp. 1-6.

[89] J. W. Booth, D. Shah, J. C. Case, E. L. White, M. C. Yuen, O. CyrChoiniere, and R. Kramer-Bottiglio, "Omniskins: Robotic skins that turn inanimate objects into multifunctional robots," Science Robotics, vol. 3, no. 22, p. eaat $1853,2018$.

[90] G. A. Pratt and M. M. Williamson, "Series elastic actuators," in Proceedings 1995 IEEE/RSJ International Conference on Intelligent Robots and Systems. Human Robot Interaction and Cooperative Robots, vol. 1, Aug 1995, pp. 399-406 vol.1.

[91] S. Kim and J. Bae, "Force-mode control of rotary series elastic actuators in a lower extremity exoskeleton using model-inverse time delay control," IEEE/ASME Transactions on Mechatronics, vol. 22, no. 3, pp. 1392-1400, June 2017.

[92] L. C. Visser, R. Carloni, and S. Stramigioli, "Energy-efficient variable stiffness actuators," IEEE Transactions on Robotics, vol. 27, no. 5, pp. 865-875, Oct 2011.

[93] B. Ugurlu, C. Doppmann, M. Hamaya, P. Forni, T. Teramae, T. Noda, and J. Morimoto, "Variable ankle stiffness improves balance control: Experiments on a bipedal exoskeleton," IEEE/ASME Transactions on Mechatronics, vol. 21, no. 1, pp. 79-87, Feb 2016.

[94] S. Wolf, G. Grioli, O. Eiberger, W. Friedl, M. Grebenstein, H. Höppner, E. Burdet, D. G. Caldwell, R. Carloni, M. G. Catalano et al., "Variable stiffness actuators: Review on design and components," IEEE/ASME transactions on mechatronics, vol. 21, no. 5, pp. 2418-2430, 2016.

[95] R. Van Ham, T. G. Sugar, B. Vanderborght, K. W. Hollander, and D. Lefeber, "Compliant actuator designs," IEEE Robotics \& Automation Magazine, vol. 16, no. 3, 2009.

[96] K. T. Park and H. M. Kim, "Wearable robotic system using hydraulic actuator," in 2011 11th International Conference on Control, Automation and Systems, Oct 2011, pp. 1697-1701.

[97] J. Choo and J. H. Park, "Increasing payload capacity of wearable robots using linear actuators," IEEE/ASME Transactions on Mechatronics, vol. 22, no. 4, pp. 1663-1673, Aug 2017.

[98] N. Sebanz, H. Bekkering, and G. Knoblich, "Joint action: bodies and minds moving together," Trends in cognitive sciences, vol. 10, no. 2, pp. 70-76, 2006.

[99] S. Nikolaidis and J. Shah, "Human-robot cross-training: Computational formulation, modeling and evaluation of a human team training strategy," in Proceedings of the 8th ACM/IEEE international conference on Human-robot interaction. IEEE Press, 2013, pp. 33-40.

[100] K. A. Loveland and S. H. Landry, "Joint attention and language in autism and developmental language delay," Journal of autism and developmental disorders, vol. 16, no. 3, pp. 335-349, 1986.

[101] T. L. Stanton-Chapman and M. E. Snell, "Promoting turn-taking skills in preschool children with disabilities: The effects of a peerbased social communication intervention," Early Childhood Research Quarterly, vol. 26, no. 3, pp. 303 - 319, 2011. [Online]. Available: http://www.sciencedirect.com/science/article/pii/S0885200610000888

[102] R. Fang, M. Doering, and J. Y. Chai, "Embodied collaborative referring expression generation in situated human-robot interaction," in Proceedings of the Tenth Annual ACM/IEEE International Conference on Human-Robot Interaction, ser. HRI ' 15 . New York, NY, USA: ACM, 2015, pp. 271-278. [Online]. Available: http://doi.acm.org/10.1145/2696454.2696467 
[103] M. M. Moniri, F. A. E. Valcarcel, D. Merkel, and D. Sonntag, "Human gaze and focus-of-attention in dual reality human-robot collaboration," in 2016 12th International Conference on Intelligent Environments (IE), Sept 2016, pp. 238-241.

[104] B. Mutlu, A. Terrell, and C.-M. Huang, "Coordination mechanisms in human-robot collaboration," in Proceedings of the Workshop on Collaborative Manipulation, 8th ACM/IEEE International Conference on Human-Robot Interaction. Citeseer, 2013.

[105] P. Liu, D. F. Glas, T. Kanda, H. Ishiguro, and N. Hagita, "It's not polite to point: generating socially-appropriate deictic behaviors towards people," in Proceedings of the 8th ACM/IEEE international conference on Human-robot interaction. IEEE Press, 2013, pp. 267274.

[106] S. C. Levinson, "Turn-taking in human communication-origins and implications for language processing," Trends in cognitive sciences, vol. 20, no. 1, pp. 6-14, 2016.

[107] G. Skantze, A. Hjalmarsson, and C. Oertel, "Turn-taking, feedback and joint attention in situated human-robot interaction," Speech Communication, vol. 65, pp. 50-66, 2014.

[108] C. Oertel, M. Włodarczak, J. Edlund, P. Wagner, and J. Gustafson, "Gaze patterns in turn-taking," in Thirteenth Annual Conference of the International Speech Communication Association, 2012.

[109] R. Sato and Y. Takeuchi, "Coordinating turn-taking and talking in multi-party conversations by controlling robot's eye-gaze." in RO-MAN, 2014, pp. 280-285.

[110] C. Park, J. Kim, and J.-H. Kang, "Turn-taking intention recognition using multimodal cues in social human-robot interaction," in Control, Automation and Systems (ICCAS), 2017 17th International Conference on. IEEE, 2017, pp. 1300-1302.

[111] P. Baxter, J. Kennedy, T. Belpaeme, R. Wood, I. Baroni, and M. Nalin, "Emergence of turn-taking in unstructured child-robot social interactions," in Human-Robot Interaction (HRI), 2013 8th ACM/IEEE International Conference on. IEEE, 2013, pp. 77-78.

[112] R. Alami, A. Clodic, V. Montreuil, E. A. Sisbot, and R. Chatila, "Toward human-aware robot task planning." in AAAI spring symposium: to boldly go where no human-robot team has gone before, 2006, pp. $39-46$.

[113] M. Cirillo, L. Karlsson, and A. Saffiotti, "Human-aware task planning: An application to mobile robots," ACM Trans. Intell. Syst. Technol., vol. 1, no. 2, pp. 15:1-15:26, Dec. 2010. [Online]. Available: http://doi.acm.org/10.1145/1869397.1869404

[114] R. Alami, M. Gharbi, B. Vadant, R. Lal-lement, and A. Suarez, "On human-aware task and motion planning abilities for a team-mate robot," in Human-Robot Collaboration for Industrial Manufacturing Workshop, RSS 2014, UC Berkeley, United States, Jul. 2014. [Online]. Available: https://hal.archives-ouvertes.fr/hal-01110191

[115] S. M. LaValle, Planning algorithms. Cambridge university press, 2006.

[116] A. Agostini, C. Torras, and F. Wörgötter, "Integrating task planning and interactive learning for robots to work in human environments." in IJCAI, 2011, pp. 2386-2391.

[117] G. Milliez, M. Warnier, A. Clodic, and R. Alami, "A framework for endowing an interactive robot with reasoning capa-bilities about perspective-taking and belief management," in The 23rd IEEE International Symposium on Robot and Human In-teractive Communication. IEEE, 2014, pp. 1103-1109.

[118] E. A. Sisbot, R. Ros, and R. Alami, "Situation assessment for humanrobot interactive object manipula-tion," in 2011 RO-MAN. IEEE, 2011, pp. 15-20.

[119] M. Vircikova, M. Pala, P. Smolar, and P. Sincak, "Neural approach for personalised emotional model in human-robot interaction," in The 2012 International Joint Conference on Neural Networks (IJCNN). IEEE, 2012, pp. 1-8.

[120] H. Mayer, F. Gomez, D. Wierstra, I. Nagy, A. Knoll, and J. Schmidhuber, "A system for robotic heart surgery that learns to tie knots using recurrent neural networks," Advanced Robotics, vol. 22, no. 13-14, pp. 1521-1537, 2008.

[121] C. Yang, X. Wang, L. Cheng, and H. Ma, "Neural-learning-based telerobot control with guaranteed perfor-mance," IEEE Transactions on Cybernetics, 2016.

[122] F. Lewis, S. Jagannathan, and A. Yesildirak, Neural network control of robot manipulators and non-linear sys-tems. CRC Press, 1998.

[123] L. Pinto and A. Gupta, "Supersizing self-supervision: Learning to grasp from 50k tries and 700 robot hours," in Robotics and Automation (ICRA), 2016 IEEE International Con-ference on. IEEE, 2016, pp. $3406-3413$.
[124] O. D. Lara and M. A. Labrador, "A survey on human activity recognition using wearable sensors." IEEE Communications Surveys and Tutorials, vol. 15, no. 3, pp. 1192-1209, 2013.

[125] M. Belkin and P. Niyogi, "Laplacian eigenmaps for dimensionality reduction and data repre-sentation," Neural computation, vol. 15, no. 6, pp. 1373-1396, 2003.

[126] K. E. Kaplan, "Improving inclusion segmentation task performance through human-intent based human-robot collaboration," in 2016 11th ACM/IEEE International Conference on Human-Robot In-teraction (HRI). IEEE, 2016, pp. 623-624.

[127] S. Xiao, Z. Wang, and J. Folkesson, "Unsupervised robot learning to predict person motion," in Robotics and Automation (ICRA), 2015 IEEE International Con-ference on. IEEE, 2015, pp. 691-696.

[128] S. Calinon and A. Billard, "Active teaching in robot programming by demonstration," in RO-MAN 2007-The 16th IEEE International Symposium on Robot and Human Interactive Communication. IEEE, 2007, pp. 702-707.

[129] J. Chen, H. Y. Lau, W. Xu, and H. Ren, "Towards transferring skills to flexible surgical robots with pro-gramming by demonstration and reinforcement learning," in 2016 Eighth International Conference on Advanced Computational Intelligence (ICACI). IEEE, 2016, pp. 378384.

[130] L. Rozo, S. Calinon, D. G. Caldwell, P. Jimnez, and C. Torras, "Learning physical collaborative robot behaviors from human demonstrations," IEEE Transactions on Robotics, vol. 32, no. 3, pp. 513-527, June 2016.

[131] X. S. Papageorgiou, G. Chalvatzaki, C. S. Tzafestas, and P. Maragos, "Hidden markov modeling of human pathological gait using laser range finder for an assisted living intelligent robotic walker," in Intelligent Robots and Systems (IROS), 2015 IEEE/RSJ Interna-tional Conference on. IEEE, 2015, pp. 6342-6347.

[132] D. Kulic and E. A. Croft, "Affective state estimation for human-robot interaction," IEEE Transactions on Robotics, vol. 23, no. 5, pp. 9911000, 2007.

[133] S. Calinon, P. Evrard, E. Gribovskaya, A. Billard, and A. Kheddar, "Learning collaborative manipulation tasks by demonstration using a haptic interface," in Advanced Robotics, 2009. ICAR 2009. International Conference on. IEEE, 2009, pp. 1-6.

[134] T. Asfour, P. Azad, F. Gyarfas, and R. Dillmann, "Imitation learning of dual-arm manipulation tasks in humanoid ro-bots," International Journal of Humanoid Robotics, vol. 5, no. 02, pp. 183-202, 2008.

[135] S. Calinon, F. Guenter, and A. Billard, "Goal-directed imitation in a humanoid robot," in Robotics and Automation, 2005. ICRA 2005. Proceedings of the 2005 IEEE International Conference on. IEEE, 2005, pp. 299-304.

[136] B. Nemec, A. Gams, M. Denia, and A. Ude, "Human-robot cooperation through force adaptation using dynamic motion primitives and iterative learning," in 2014 IEEE International Conference on Robotics and Biomimetics (ROBIO 2014), Dec 2014, pp. 1439-1444.

[137] H. B. Amor, G. Neumann, S. Kamthe, O. Kroemer, and J. Peters, "Interaction primitives for human-robot cooperation tasks," in 2014 IEEE International Conference on Robotics and Automation (ICRA), May 2014, pp. 2831-2837.

[138] M. Prada, A. Remazeilles, A. Koene, and S. En-do, "Dynamic movement primitives for human-robot interaction: compari-son with human behavioral observation," in 2013 IEEE/RSJ International Conference on Intelligent Robots and Systems. IEEE, 2013, pp. 1168-1175.

[139] L. P. Kaelbling, M. L. Littman, and A. W. Moore, "Reinforcement learning: A survey," Journal of artificial intelligence research, vol. 4, pp. 237-285, 1996.

[140] Y. Gu, A. Thobbi, and W. Sheng, "Human-robot collaborative manipulation through imitation and rein-forcement learning," in Information and Automation (ICIA), 2011 IEEE International Conference on. IEEE, 2011, pp. 151-156.

[141] R. Hafner and M. Riedmiller, "Neural reinforcement learning controllers for a real robot appli-cation," in Robotics and Automation, 2007 IEEE International Conference on. IEEE, 2007, pp. 2098-2103.

[142] F. Dimeas and N. Aspragathos, "Reinforcement learning of variable admittance control for human-robot co-manipulation," in 2015 IEEE/RSJ International Conference on Intelligent Robots and Systems (IROS), Sept 2015, pp. 1011-1016.

[143] S. Haddadin, A. Albu-Schaffer, A. De Luca, and G. Hirzinger, "Collision detection and reaction: A contribution to safe physical humanrobot interaction," in 2008 IEEE/RSJ International Conference on Intelligent Robots and Systems. IEEE, 2008, pp. 3356-3363.

[144] G. B. Avanzini, N. M. Ceriani, A. M. Zanchet-tin, P. Rocco, and L. Bascetta, "Safety control of industrial robots based on a distributed 
dis-tance sensor," IEEE Transactions on Control Systems Technology, vol. 22, no. 6, pp. 2127-2140, 2014.

[145] Y. K. Hwang and N. Ahuja, "Gross motion planninga survey," $A C M$ Computing Surveys (CSUR), vol. 24, no. 3, pp. 219-291, 1992.

[146] P. Ogren, N. Egerstedt, and X. Hu, "Reactive mobile manipulation using dynamic trajectory tracking," in Robotics and Automation, 2000. Proceedings. ICRA'OO. IEEE In-ternational Conference on, vol. 4. IEEE, 2000, pp. 3473-3478.

[147] S. Hirche and M. Buss, "Human-oriented control for haptic teleoperation," Proceedings of the IEEE, vol. 100, no. 3, pp. 623-647, March 2012 .

[148] H.-D. Lee, B.-K. Lee, W.-S. Kim, J.-S. Han, K.-S. Shin, and C.-S. Han, "Humanrobot cooperation control based on a dynamic model of an upper limb exoskeleton for human power amplification," Mechatronics, vol. 24, no. 2, pp. 168 - 176, 2014. [Online]. Available: http://www.sciencedirect.com/science/article/pii/S0957415814000087

[149] K. Kiguchi and Y. Hayashi, "An emg-based control for an upper-limb power-assist exoskeleton robot," IEEE Transactions on Systems, Man, and Cybernetics, Part B (Cybernetics), vol. 42, no. 4, pp. 1064-1071, Aug 2012.

[150] L. Zhao, Y. Liu, K. Wang, P. Liang, and R. Li, "An intuitive human robot interface for tele-operation," in 2016 IEEE International Conference on Real-time Computing and Robotics (RCAR), June 2016, pp. 454-459.

[151] Z. Li, B. Wang, F. Sun, C. Yang, Q. Xie, and W. Zhang, "semg-based joint force control for an upper-limb power-assist exoskeleton robot," IEEE Journal of Biomedical and Health Informatics, vol. 18, no. 3, pp. 1043-1050, May 2014.

[152] R. R. Burridge and K. A. Hambuchen, "Using prediction to enhance remote robot supervision across time delay," in 2009 IEEE/RSJ International Conference on Intelligent Robots and Systems, Oct 2009, pp. $5628-5634$.

[153] J. R. Medina, M. Lawitzky, A. Molin, and S. Hirche, "Dynamic strategy selection for physical robotic assistance in partially known tasks," in 2013 IEEE International Conference on Robotics and Automation, May 2013, pp. 1180-1186.

[154] P. Evrard and A. Kheddar, "Homotopy switching model for dyad haptic interaction in physical collaborative tasks," in World Haptics 2009 - Third Joint EuroHaptics conference and Symposium on Haptic Interfaces for Virtual Environment and Teleoperator Systems, March 2009, pp. 45-50.

[155] Y. Li, K. P. Tee, W. L. Chan, R. Yan, Y. Chua, and D. K. Limbu, "Continuous role adaptation for human-robot shared control," IEEE Transactions on Robotics, vol. 31, no. 3, pp. 672-681, June 2015.

[156] P. A. Hancock, D. R. Billings, K. E. Schaefer, J. Y. Chen, E. J. De Visser, and R. Parasuraman, "A meta-analysis of factors affecting trust in human-robot interaction," Human Factors, vol. 53, no. 5, pp. 517-527, 2011.

[157] S. Nikolaidis and J. Shah, "Human-robot teaming using shared mental models," ACM/IEEE HRI, 2012.

[158] M. Scheutz, "Computational mechanisms for mental models in humanrobot interaction," in International Conference on Virtual, Augmented and Mixed Reality. Springer, 2013, pp. 304-312.

[159] P. Corke, Robotics, Vision and Control: Fundamental Algorithms In MATLAB® Second, Completely Revised. Springer, 2017, vol. 118.

[160] S. Rea, "Advances to Brain-Interface Technology Provide Clearer Insight into Visual System Than Ever Before ." https://www.cmu.edu/dietrich/news/newsstories/2017/december/neuroscientists-engineers-new-eeg.html accessed: 2017-01-07.

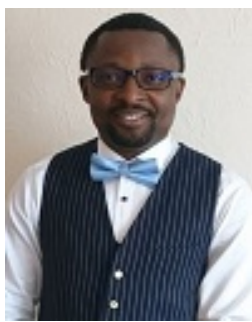

Uchenna Emeoha Ogenyi received the B.Eng. degree in Computer Engineering from Enugu State University of Science and Technology, Nigeria, in 2007 and MSc. degree in Computer Systems and Software Engineering from the University of Greenwich, UK in 2014. He is currently a PhD candidate in the School of Computing at the University of Portsmouth, UK. His research interest includes machine learning and its applications in human-robot interaction/collaboration.

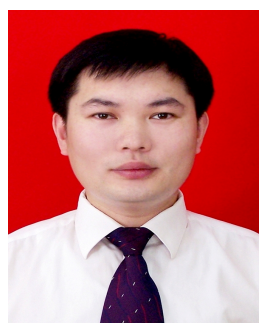

Jinguo Liu (M'07-SM'18) received the Ph.D. degree in mechatronics from the Shenyang Institute of Automation (SIA), Chinese Academy of Sciences (CAS), in 2007, where he has been a Full Professor, since January 2011, has also been the Assistant Director of the State Key Laboratory of Robotics, since 2008, and has also been the Associate Director of the Center for Space Automation Technologies and Systems, since 2015. His research interests include bio-inspired robotics and space robot. $\mathrm{He}$ has authored or coauthored three books, over 100 articles and holds 50 patents in above areas.

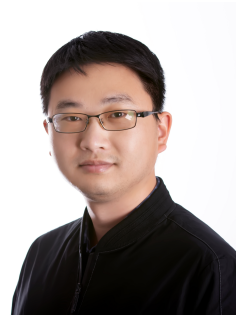

Chenguang Yang (M'10-SM'16) is a Professor of Robotics with University of the West of England. $\mathrm{He}$ received the $\mathrm{Ph} . \mathrm{D}$. degree in control engineering from the National University of Singapore, Singapore, in 2010 and performed postdoctoral research in human robotics at Imperial College London, London, UK from 2009 to 2010 . He has been awarded EU Marie Curie International Incoming Fellowship, UK EPSRC UKRI Innovation Fellowship, and the Best Paper Award of the IEEE Transactions on Robotics as well as over ten conference Best Paper Awards. His research interest lies in human robot interaction and intelligent system design.

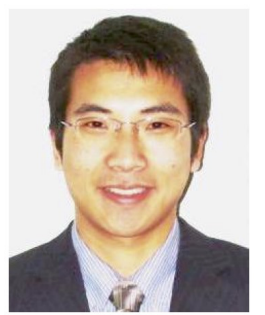

Zhaojie Ju (M'08-SM'16) received the B.S. in automatic control and the M.S. in intelligent robotics both from Huazhong University of Science and Technology, China, and the Ph.D. degree in intelligent robotics at the University of Portsmouth, UK.

He held a research appointment at the University College London, London, U.K., before he started his independent academic position at the University of Portsmouth, U.K., in 2012. His research interests include machine intelligence, pattern recognition, and their applications on human motion analysis, multi-fingered robotic hand control, humanrobot interaction and collaboration, and robot skill learning. He has authored or co-authored over 170 publications in journals, book chapters, and conference proceedings and received four Best Paper Awards and one Best AE Award in ICRA2018.

Dr. Ju is an Associate Editor of the IEEE TRANSACTIONS ON CYBERNETICS, Journal of Intelligent \& Fuzzy Systems, International Journal of Fuzzy Systems, and Chinese Journal of Mechanical Engineering.

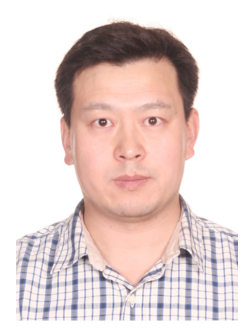

Honghai Liu received the Ph.D. degree in robotics from Kings College London, London, U.K., in 2003.

$\mathrm{He}$ joined the University of Portsmouth, Portsmouth, U.K., in September 2005. He previously held the position of research appointments with the Universities of London and Aberdeen, as well as Project Leader appointments in the large-scale industrial control and system integration industry. His current research interests include biomechanics, intelligent sensing, pattern recognition, intelligent video analytics, and wearable robotics, and their practical applications with an emphasis on approaches that could make contributions to the intelligent connection of perception to action using contextual information. He has published numerous peer-reviewed international journal and conference papers and received four best paper awards. Prof. Liu is an Associate Editor of the IEEE TRANSACTIONS ON INDUSTRIAL ELECTRONICS. 